\title{
Missed Diagnosis
}

\section{Pyogenic liver abscess with two normal ultrasound scans}

\author{
R.G. Bunney \\ Department of Infectious and Tropical Diseases, East Birmingham Hospital, Bordesley Green East, \\ Birmingham, UK.
}

\begin{abstract}
Summary: I report a case of a large liver abscess causing a severe debilitating condition in which the diagnosis was delayed by several weeks following two normal ultrasound scans at days 5 and 19 of the illness.

Up to one in four cases of liver abscess can be missed on ultrasound. A high degree of clinical suspicion must be maintained and repeated or alternative imaging methods used if the diagnosis of this rare, curable but often fatal condition is not to be missed.
\end{abstract}

\section{Introduction}

Pyogenic liver abscess is associated with a high mortality of up to $40 \%$ despite the availability of effective therapy, often because of delay in diagnosis. $^{1,2}$ This delay is partly due to its characteristically non-specific clinical presentation and to its low prevalence rate, estimated to be around one case per year in an average general hospital in the UK. ${ }^{3}$ Many cases are only diagnosed at post-mortem after the patient has been assumed to have disseminated malignant disease (accounting for 5 of 13 cases in one series) and further investigations have been withheld. ${ }^{1}$

Perera et al. ${ }^{4}$ in a review of liver abscesses seen at the Royal Free Hospital over a 9-year period, concluded that ultrasonography has superseded isotopic liver scanning in the initial screening for liver abscess. This case emphasizes that even if liver abscess has been considered, the correct diagnosis may be considerably delayed if repeated normal ultrasound scans are assumed to have excluded the condition.

\section{Case report}

A 40 year old Irish male was admitted with a 3-day history of generalized abdominal discomfort, vomiting and diarrhoea. On examination the only

Correspondence: R.G. Bunney, B.Sc., M.R.C.P., Unit B, Stobhill General Hospital, Glasgow G21 3UW, UK.

Accepted: 3 December 1987 abnormalities were a fever of $37.8^{\circ} \mathrm{C}$ and diffuse abdominal tenderness. Initial haematological and biochemical investigations were unremarkable apart from an (ESR) of $30 \mathrm{~mm} / \mathrm{h}$. A provisional diagnosis of gastroenteritis was made, adequate oral fluid replacement given and stool samples sent for microscopy and culture.

Two days after admission the vomiting and diarrhoea had stopped but the patient remained unwell with a pyrexia of $38.9^{\circ} \mathrm{C}$, tenderness in the right hypochondrium and crepitations heard over the right lower zone of the chest. A diagnosis of liver abscess was considered. At this stage a chest radiograph was normal, blood count and biochemistry remained normal, stool microscopy showed a few white cells only and culture revealed no pathogens; blood cultures were sterile. Ultrasonography of the liver by a consultant radiologist using a Siemans RAI machine on day 5 of the illness showed a normal homogeneous pattern. The patient gradually improved without further therapy and on day 10 was discharged having been apyrexial for 2 days.

On day 16 he was readmitted with rigors, bilateral loin pain and weight loss. On examination the liver was slightly tender and palpable extending $2 \mathrm{~cm}$ below the costal margin. There was a fever of $39^{\circ} \mathrm{C}$. Investigations included haemoglobin $12.5 \mathrm{~g} / \mathrm{dl}$ and white cell count $13 \times 10^{9} / 1(80 \%$ neutrophils); ESR was $90 \mathrm{~mm} / \mathrm{h}$, serum alkaline phosphatase and aspartate transaminase were now slightly raised. Blood cultures were sterile. Repeat ultrasonography of the liver on day 19 of the illness by the same

(C) The Fellowship of Postgraduate Medicine, 1988 
operator was normal, as was intravenous urography. Mantoux test (1 unit PPD) was positive with $5 \mathrm{~mm}$ induration. A percutaneous liver biopsy showed non-specific reactive hepatitis; bone marrow aspirate and trephine showed increased iron stores compatible with chronic disease. A therapeutic trial of antituberculous chemotherapy with isoniazid and ethambutol was commenced and abdominal computed tomography (CT) arranged.

This CT scan on day 40 of the illness showed a large abscess in the left lobe of the liver. A further ultrasound scan of the liver showed a large area of abnormal echoes in the left lobe, aspiration of which produced toffee smelling pus. A catheter left in situ drained 2 litres of the same material in $48 \mathrm{~h}$, culture of which grew Streptococcus milleri and Actinomyces myeri. The patient responded well to benzylpenicillin and the drain was removed after 10 days. He was discharged taking oral clindamycin to complete a total of 4 weeks antibacterial chemotherapy during which time all his symptoms resolved.

\section{Discussion}

Liver abscess characteristically presents in a nonspecific way and should be considered in all cases of pyrexia of unknown origin. Initial investigations frequently only show slight anaemia, mild elevation of erythrocyte sedimentation rate and alkaline phosphatase levels with slight fall in serum albumin. In this patient, ultrasound scanning of the liver failed to detect any abnormality of the liver at 5 and 19 days of an illness which later proved to be due to liver abscess. Cunningham ${ }^{5}$ has shown that material rich in protein and lipid from a liver abscess can be echogenic and thus misdiagnosed as a solid mass or even overlooked if the echodensity was similar to surrounding tissue. This may explain our findings and it is of note that the third

\section{References}

1. Northover, J.M.A., Jones, B.J.N., Dawson, J.L. \& Williams, $R$. Difficulties in the diagnosis and management of pyogenic liver abscess. Br J Surg 1982, 69: 48-51.

2. Satiani, B. \& Davidson, E.D. Hepatic abscesses: improvement in mortality with early diagnosis and treatment. Am J Surg 1978, 135: 647-650.

3. Young, A.E. The clinical presentation of pyogenic liver abscess. Br J Surg 1976, 63: 216-219.

4. Perera, M.R., Kirk, A. \& Noone, P. Presentation, diagnosis and management of liver abscess. Lancet 1980, ii: 629-632. ultrasound scan showed a large area of abnormal echoes which was not frankly fluid and could equally have been a solid mass.

Both isotopic and ultrasound techniques have been reported to have similar sensitivities of 75$88 \%$ for the diagnosis of liver abscess. ${ }^{1,6,7}$

False negatives using one technique may be picked up by the other ${ }^{4,6}$ but the commonest reasons for negative diagnoses with both techniques are lesions less than $2 \mathrm{~cm}$ in diameter and multiple small abscesses. ${ }^{4,6}$ These lesions accounted for $12 \%$ and $5 \%$ of cases of liver abscess in two series. ${ }^{6.7}$

CT scanning has been shown to detect up to $95 \%$ of lesions but also fails to detect lesions less than $2 \mathrm{~cm}$ in diameter. ${ }^{7}$ It is more likely to detect other conditions presenting in a similar manner such as lymphoma confined to the abdominal nodes. Other well documented causes of false negative examinations using ultrasound include fatty infiltration causing a markedly echogenic liver $\mathrm{O}$ and lesions high in the dome of the liver, a difficult area to evaluate sonographically. ${ }^{6}$

Ultrasound scanning is more likely than isotope 음 scanning to reveal the structure of detected lesions and since it can also assist in placement of a needle to aspirate pus (thus confirming the diagnosis and aiding treatment) it should be the screening method of choice. Should it be negative, isotope lives scanning or CT scanning should be performes. More importantly all techniques miss early sma lesions and should be repeated at a later date $\overrightarrow{\text { if }}$ negative.

Most importantly a high degree of clinical suspicion should be maintained if cases of this potentially fatal illness, which is almost always curable, are to be detected.

\section{Acknowledgement}

I thank Dr M.J. Wood for permission to report this case.

5. Cunningham, J.J. In vitro gray scale echography of protein-lipid fluid collection in liver tissue. JCU 1976, N 14: 255-258.

6. Rubinson, H.A., Isikoff, M.B. \& Hill, M.C. Diagnostic N imaging of hepatic abscesses: a retrospective analysis. $\mathfrak{\omega}$ AJR 1980, 135: 735-740.

7. Mathieu, D., Vasile, N., Fangniez, P.L., Segui, S., Graby, D. \& Lorde, D. Dynamic CT features of hepatic abscesses. Radiology 1985, 154: 749-752. 